\title{
Renal Artery Embolism: Prospective Study of 41 Patients Based on a Diagnostic and Therapeutic Algorithm
}

\author{
Joan Fort ${ }^{*}, 1$, Alfons Segarra ${ }^{1}$, Manel Matas ${ }^{2}$, Antonio Segarra $^{3}$ and Joaquim Camps ${ }^{1}$ \\ ${ }^{1}$ Department of Nephrology, ${ }^{2}$ Department of Vascular Surgery and ${ }^{3}$ Department of Interventional Angiography, \\ Hospital General Universitari Vall d'Hebron, Universitat Autónoma, Barcelona, Spain
}

\begin{abstract}
Renal artery embolism (RAE) is an underdiagnosed condition leading to acute renal failure in patients with a single functioning kidney. We prospectively studied 41 patients according to a previously validated algorithm based on Lactate Dehydrogenase (LDH) determination, which enables us to identify RAE patients and allocate them to a different protocolled treatment.

The most frequent symptom was atypical low back pain. Atrial fibrillation was present in $65.8 \%$ of patients. The most frequent site of the embolism was the main renal artery of a single kidney. Surgery was performed in 13 patients, fibrinolytic treatment in 17 and anticoagulation in 11. Mean LDH levels were $1690 \pm 1108 \mathrm{U} / \mathrm{L}$. Oliguria was present in 15 patients. Hemodialysis requirements were not different between patients with main RAE or intrarenal embolism, or according to treatment group.

Conclusions: Our results indicate that the use of a diagnostic algorithm based on LDH values is useful for identifying RAE patients. Further randomized trials are needed to compare results on treatment.
\end{abstract}

\section{INTRODUCTION}

Renal artery embolism (RAE) involves diagnostic and therapeutic problems that still remain unresolved. First, post mortem studies have shown that RAE is an underdiagnosed pathology [1]. It is suspected in patients with low back pain, which may or may not be associated with renal failure, and certain risk factors, such as emboligenic heart disease, atrial fibrillation, or aortic atheromatosis [2]. Nevertheless, when the clinical presentation is atypical and there are no evident associated risk factors at the time of the acute episode, the embolism can remain undetected if the diagnosis is not considered systematically.

Second, the appropriate type of treatment for each situation is controversial. Since the publication of the first reference to renal embolic disease in the related literature [3] several case series have been reported in descriptive, mainly retrospective studies, in which the patients are treated without predefined criteria [4-8]. No data are available from prospective studies in which patients are treated according to the same criteria.

The present study describes the clinical application of a previously validated diagnostic algorithm for renal artery embolism and the long-term follow-up of a prospective cohort of patients with this condition, treated according to a single therapeutic protocol.

\section{MATERIALS AND METHODOLOGY}

During the period of April 1992 to April 2004, a prospective follow-up was performed in all patients diagnosed with

*Address correspondence to this author at the Department of Nephrology, Hospital General Universitari Vall d'Hebron, Universitat Autónoma, $\mathrm{P}^{\circ}$ Vall D'Hebron 119-129, 08035, Barcelona, Spain; Tel: +34934893000, Ext. 6476; Fax: + 34 932746204; E-mail: 9159jfr@comb.es
RAE in the emergency room of a third-level referral hospital with a catchment population of approximately 500.000 inhabitants. The diagnosis of RAE was made according to a previously validated algorithm by our group based on emergency lactate dehydrogenase $(\mathrm{LDH})$ determination and the presence of clinical risk factors [9] (Fig. 1). In all cases, the diagnosis of renal embolism was confirmed by CT angiography and/or selective renal arteriography.

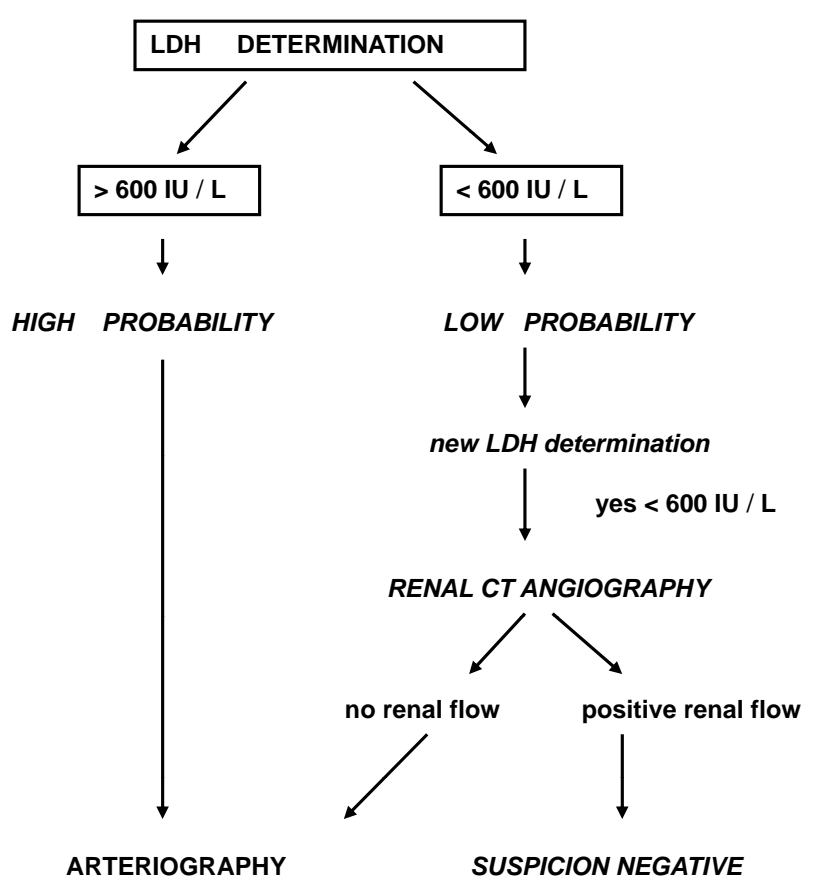

Fig. (1). Diagnostic algorithm by LDH determination.

All patients underwent electrocardiography and transthoracic or transesophageal echocardiography to determine the 
origin of the embolism and to rule out the presence of valvular disease and dilated myocardial disease.

\section{Treatment}

After the diagnosis had been established, all patients were allocated to one of the following treatment groups according to the location of the embolism and whether or not there was a single functioning kidney (Fig. 2).

\section{Surgical Embolectomy}

Patients with embolism of the main renal artery without major contraindications for surgery.

The surgical procedure consisted in renal artery arteriotomy without clamping of the aorta, followed by embolectomy with assessment of retrograde flow and intra-arterial administration of a bolus of 300.000 units of urokinase (UK) in saline solution.

Contraindications for surgical embolectomy:

- More than 85 years of age.

- Hemodynamic instability or circulatory shock with a mean arterial tension less than $100 \mathrm{mmHg}$.

- $\quad$ Severe heart failure, NYHA functional class III-IV

- Coagulation alterations; thrombopenia, less than 50.000 platelets per $\times 10 \mathrm{E} 9 / \mathrm{L}$.

- Contraindication for anesthesia as assessed by the anesthesiologist, and severe respiratory failure.

\section{Fibrinolytic Agents}

Fibrinolytic treatment was indicated in patients with a single kidney, intrarenal embolisms and no contraindications for systemic fibrinolysis, and in patients with embolism of the main renal artery (with or without concomitant intrarenal embolisms) and contraindications for surgery.

Patients were administered an intra-arterial bolus of 250.000 units of UK through a catheter placed in the main renal artery, followed by perfusion of $1000 \mathrm{U} / \mathrm{Kg}$ weight/hour of UK for 24 hours. If images of thrombosis persisted at the 24-hour radiological control, the fibrinolytic perfusion was maintained an additional 24 hours. If the thrombus had lysed, the catheter was removed and sodium heparin was given for 24 hours by perfusion pump.
Contraindications for fibrinolytic treatment (The exclusion criteria for fibrinolytic therapy are those described in the Consensus and Development Conference of 1980):

- Absolute contraindication: active hemorrhage, recent cerebral vascular accident or active intracranial process.

- Major relative contraindication: major surgery, postpartum, recent puncture biopsy of any organ, recent gastrointestinal bleeding or severe arterial hypertension.

- Minor relative contraindication: recent cardiopulmonary resuscitation maneuvers, presence of left heart mural thrombi, bacterial endocarditis, more than 75 years of age and hemorrhagic retinopaty.

\section{Anticoagulation Treatment}

Group 1: Patients with two kidneys, unilateral renal embolism, and normal renal function.

Group 2: Patients with contraindications for surgery and fibrinolytic treatment.

These cases were treated according to non-interventional criteria and received a prophylactic anticoagulation regimen to prevent future embolisms.

\section{Outcome Assessment}

The results obtained were evaluated from two perspectives:

a) Clinical outcome-defined by evidence of improved renal function, as assessed by glomerular filtration in the affected kidney, which was measured by Tc-99mDTPA isotopic renography at 8 weeks after revascularization.

b) Technical outcome-defined as revascularization of the renal arteries, as determined by selective renal arteriography or CT angiography.

\section{Statistics}

Student t-test was used to compare two means and analysis of variance followed by Student t-test with the Bonferroni correction for repeated comparison was used to compare more than two means. Dependence between quantitative variables was assessed by Pearson correlation test.
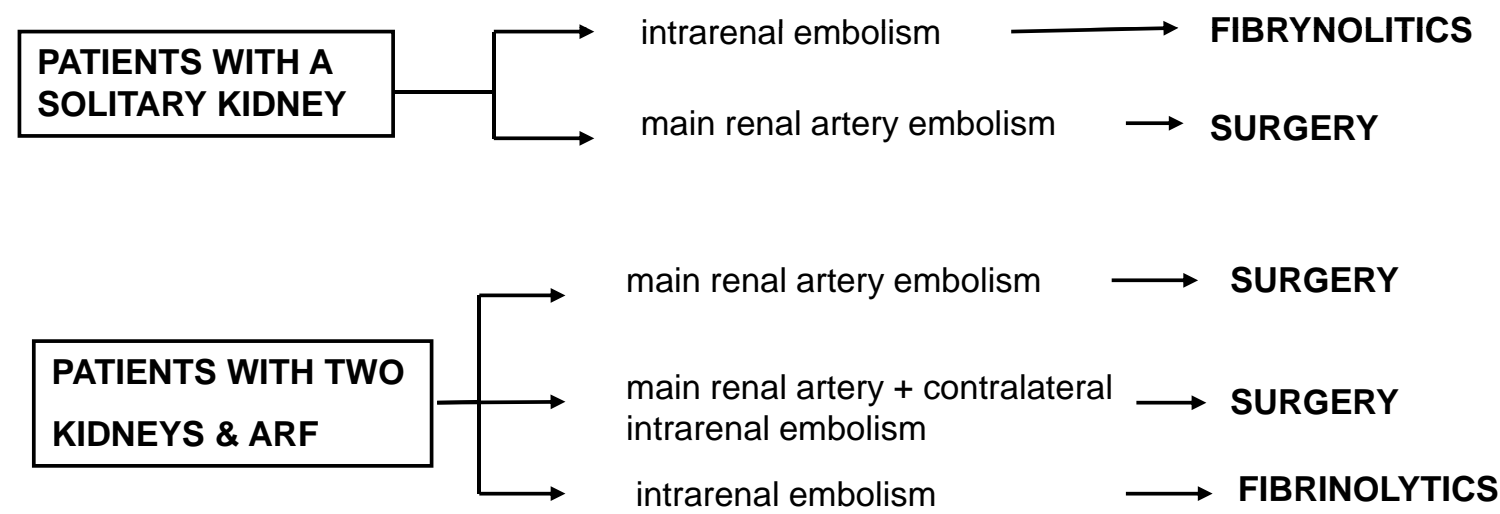

Fig. (2). Therapeutic protocol. ARF: acute renal failure. 


\section{RESULTS}

A total of 41 cases of RAE were diagnosed over the study period. The main demographic and clinical data of the patients are summarized in Table $\mathbf{1}$. The most frequent reason for the initial consultation $(85.3 \%)$ was atypical low back pain, accompanied by non-specific abdominal pain in 11 patients and macroscopic hematuria in 3 patients. The mean interval between the onset of symptoms and the diagnosis were 84 hours. Sixteen patients $(39 \%)$ had gone to the emergency room of other centers on one or more occasions over the days prior to the diagnosis because of low back pain $(n=6)$ or abdominal pain $(n=10)$. The most frequent origin of the embolism was the atrium in 29 patients $(70.7 \%)$, followed by a valvular origin, aneurysm, ventricular thrombosis and renal artery thrombosis in 1 patient each $(2.4 \%$ each), and an indeterminate location in $8(19.50 \%)$. Atrial fibrillation was present in 29 patients $(70,1 \%)$ From these, only $31 \%$ of patients were under anticoagulant treatment at the time of the embolic episode. The location of the embolism is summarized in Table 2. The most frequent site was in the main renal artery in patients with a solitary kidney of a single kidney (46.6\%). Previous embolic episodes of different localizations were reported in 10 patients $(22 \%)$ Mean levels of LDH were $1690 \mathrm{U} / \mathrm{L}$, being higher than $600 \mathrm{U} / \mathrm{L}$ in all patients. Mean serum creatinine levels were $353,6 \mu \mathrm{mol} / \mathrm{l}$. Oliguria was detected in 15 patients $(35,6 \%)$. Of these $39 \%$ had a solitary functioning kidney.

Table 1. Clinical and Laboratory Variables

\begin{tabular}{|l|lc|}
\hline \multicolumn{1}{|c|}{ Age } & \multicolumn{2}{|c|}{$66 \pm \mathbf{1 4}$} \\
\hline \hline $\begin{array}{l}\text { Total episodes } \\
\text { Gender }\end{array}$ & $\begin{array}{l}41 \\
21 \text { women } \\
20 \text { men }\end{array}$ \\
\hline History valvular disease & $21 / 41$ \\
\hline Low back pain & $35 / 41$ \\
\hline Atrial fibrillation ( AF) & $29 / 41$ \\
\hline Anticoagulation in patients with AF & $9 / 31$ \\
\hline Prior embolisms & $10 / 41$ \\
\hline Time between symptoms and diagnosis & $84 \pm 78$ hours \\
\hline Patients with oliguria & $15 / 41$ \\
\hline Creatinine $\mu$ mol/1 & $353.6 \pm 265.2$ \\
\hline $\begin{array}{l}\text { LDH U/L } \\
\text { LDH }>600 \text { U/L + diagnostic Arteriography }\end{array}$ & $1690 \pm 1108$ \\
\hline
\end{tabular}

AF: Atrial fibrillation.

Surgery was performed in 13 patients, fibrinolytic treatment in 17, and anticoagulation in 11 patients.

Figs. (3-6) show images before and after systemic fibrinolytic treatment of two different patients, one suffering from partial emboli occlusion of main renal artery and the other, multiple segmentary intrarenal emboli.

The clinical characteristics of the patients, as well as the technical results, clinical outcome, complications and mortality in each group are shown in Table 3. The group of patients treated with anticoagulation included 2 patients in whom the embolism occurred in the acute phase of a myo- cardial infarction and 3 patients in whom it occurred as a complication of dilated myocardial disease with poor systolic function. The presence of mitral valvular disease was significantly more frequent in the surgical and fibrinolytic groups as compared to the anticoagulation group. The incidence of complete arrhythmia due to atrial fibrillation at the time of the embolism episode was similar in the three groups $(P=.12)$.

\section{Table 2. Location of Embolism}

\begin{tabular}{|ll|cr|}
\hline Main renal artery & unilateral & $46.6 \%$ & 19 \\
\hline Intrarenal artery & unilateral & $22.2 \%$ & 9 \\
\hline Main renal artery & bilateral & $4.9 \%$ & 2 \\
\hline Intrarenal artery & bilateral & $2.4 \%$ & 1 \\
\hline Main renal + intrarenal artery & unilateral & $14.4 \%$ & 6 \\
\hline Main renal + intrarenal artery & bilateral & $7.1 \%$ & 3 \\
\hline Main renal + intrarenal artery & contralateral & $2.4 \%$ & 1 \\
\hline
\end{tabular}

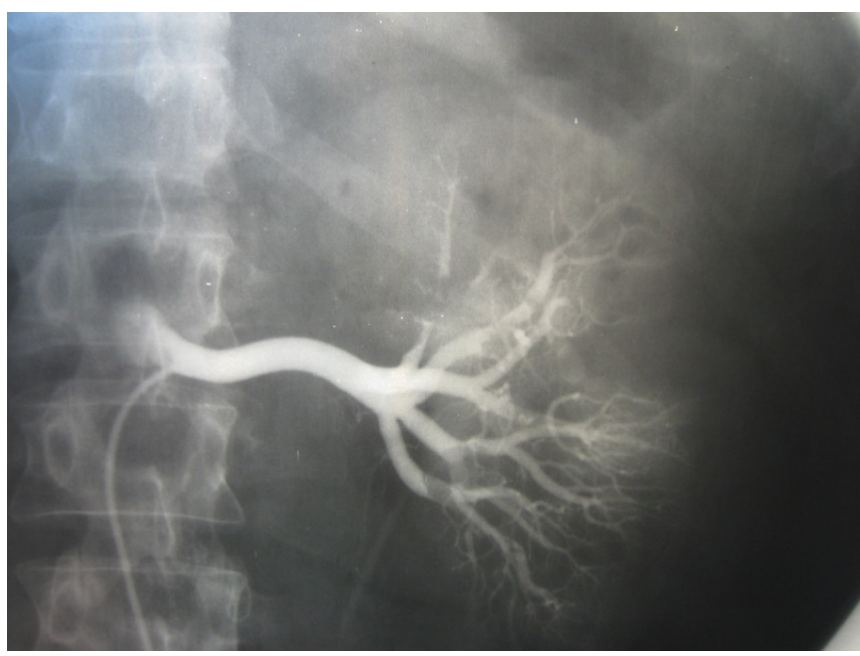

Fig. (3). Selective arteriography of a right renal artery showing partially emboli occlusion at bifurcation of the main renal artery.

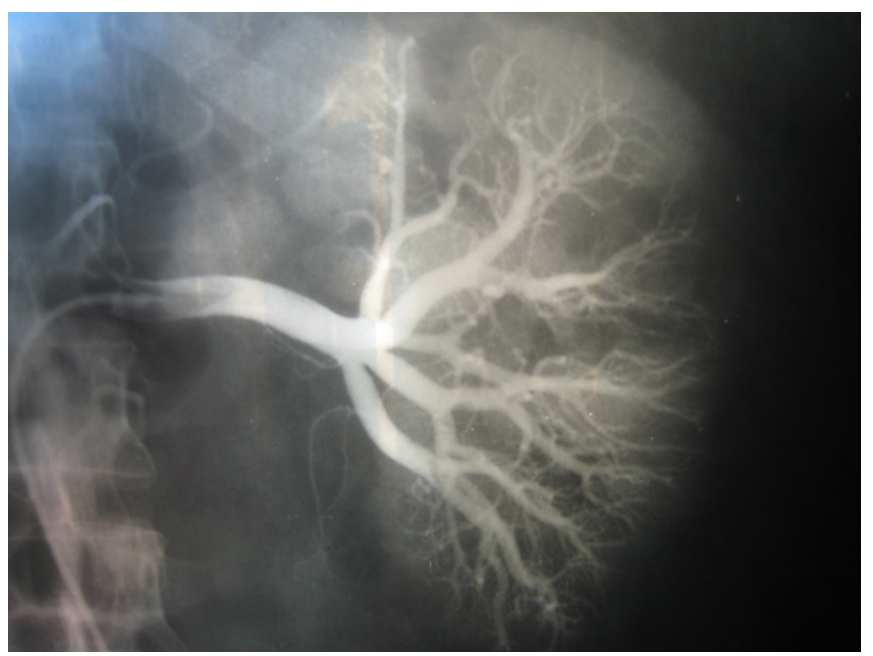

Fig. (4). Total emboli lysis after 24 hours of systemic fibrinolytic treatment. 


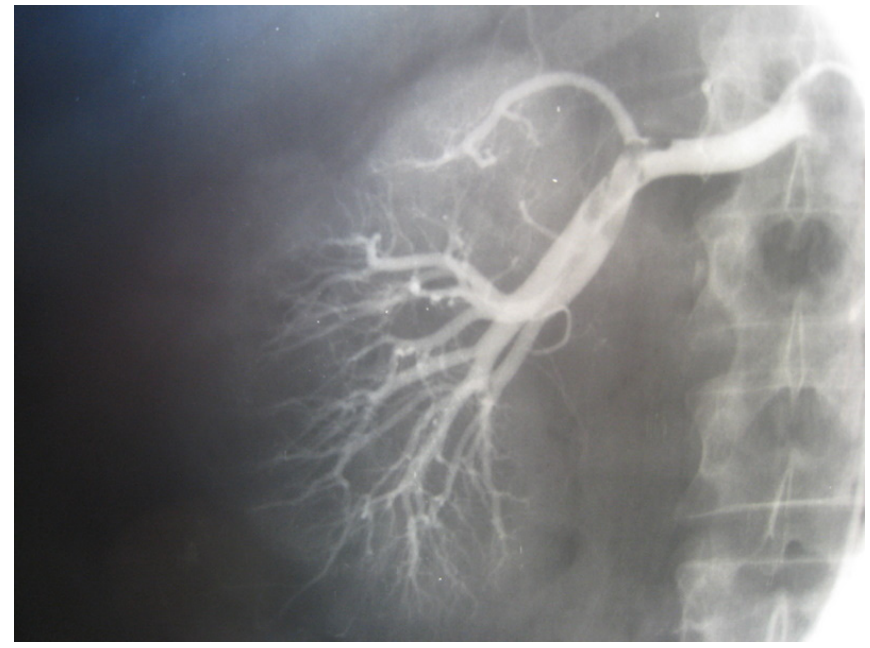

Fig. (5). Left selective renal arteriography showing multiple segmentary intrarenal emboli.

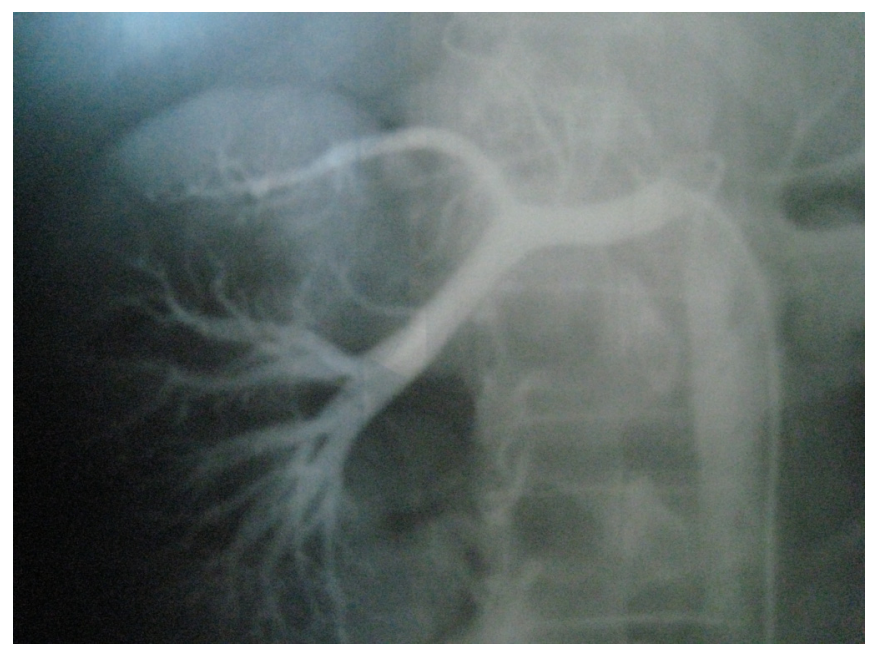

Fig. (6). Emboli lysis after fibrinolytic treatment with partial recuperation of perfusion of the parenchyma.

Ten of the 27 patients with embolism of the main renal artery $(37.03 \%)$ versus $3 / 14(21.4 \%)$ patients with intrarenal embolism required hemodialysis treatment during the acute phase $(P=.68)$. By treatment group, $8 / 13(46.5 \%)$ patients in the surgical group, $2 / 17(13.3 \%)$ in the fibrinolytic group and $3 / 11(27.3 \%)$ in the anticoagulation group required hemodialysis during the acute phase of embolism $(P=.12)$.
Finally, among these patients renal function did not recovered in eight patients, which required permanent renal replacement therapy.

\section{Complications}

The frequency and type of complication, and the mortality observed in each group are described in Table 3. The complications were qualitatively different in the various groups, but there were no significant differences as to frequency: surgical embolectomy $(n=9 ; 69.2 \%)$ versus fibrinolytic therapy $(n=3 ; 17.6 \%)$ and anticoagulation $(n=5$; 45.4\%) $(P=.6)$.

All the bleeding complications observed $(n=6 ; 14.6 \%)$ occurred in patients with embolism of the main renal artery. Three cases involved postoperative bleeding and 3 others were associated with intra-arterial fibrinolytic treatment lasting more than 48 hours because patency had not been documented on radiologic monitoring at 24 and 36 hours.

Overall mortality was $14.6 \%, 2$ patients in the surgical group $(15.4 \%)$ and 6 patients in the anticoagulation group $(36.4 \%)$. The causes of death were sepsis $(n=3)$, massive mesenteric embolism $(n=1)$ and multiorgan failure $(n=2)$.

\section{DISCUSSION}

This study is the first to describe the clinical experience of a large prospective series of patients with renal artery embolism, diagnosed with a previously validated algorithm and treated in a protocolled manner according to established criteria. The clinical data regarding the presence of risk factors for embolism, characteristics of the acute episode, and location of the emboli were similar to the reported data for another series $[6,8]$, with the exception of the origin of the emboli. Our series had a higher percentage of atrial origin, which may be attributable to the fact that transthoracic or transesophageal echocardiography were used to investigate the origin of the embolus in all cases. Our data are also in keeping with the recently reported underuse of anticoagulants in patients with atrial fibrillation [10].

The first finding of interest from the present study is the confirmation that systematic determination of serum $\mathrm{LDH}$ levels in patients consulting for atypical low back or abdominal pain is highly useful clinically, even when there are no evident emboligenic risk factors. Elevated serum LDH concentration has been described as a sensitive marker of tissue infarction $[9,11]$ (acute myocardial infarction, renal

Table 3. Treatment Outcome, Complications and Mortality

\begin{tabular}{|c|c|c|c|c|c|c|c|c|c|c|c|c|c|}
\hline Group & $\mathbf{N}^{0}$ & Age & $\begin{array}{c}\mathbf{L D H} \\
\mathbf{U} / \mathbf{L}\end{array}$ & $\begin{array}{l}\text { Hist } \\
\text { Emb }\end{array}$ & $\mathbf{A F}$ & Valvul & $\begin{array}{c}\text { Creat } \\
\mu \mathrm{mol} / \mathrm{l}\end{array}$ & $\begin{array}{l}\text { Clinical } \\
\text { Results }\end{array}$ & $\begin{array}{c}\text { Technical } \\
\text { Results }\end{array}$ & $\begin{array}{l}\text { Hemato } \\
\text { Hemorr }\end{array}$ & Sepsis & Shock & Death \\
\hline Surgery & 13 & $67.9 \pm 12$ & $1900 \pm 847$ & $15 \%$ & $\begin{array}{c}10 \\
(77 \%)\end{array}$ & $61 \%$ & $424.32 \pm 150.28$ & $\begin{array}{c}5 \\
(38.4 \%)\end{array}$ & $13(100 \%)$ & $\begin{array}{c}3 \\
(23 \%)\end{array}$ & $\begin{array}{c}1 \\
(7.69 \%)\end{array}$ & $\begin{array}{c}5 \\
(38.4 \%)\end{array}$ & $\begin{array}{c}2 \\
(15.3 \%)\end{array}$ \\
\hline Fibrinol & 17 & $63.1 \pm 14$ & $1513 \pm 1074$ & $31 \%$ & $\begin{array}{c}12 \\
(70 \%)\end{array}$ & $56 \%$ & $344.76 \pm 89.28$ & $\begin{array}{c}12 \\
(70 \%)\end{array}$ & $15(75 \%)$ & $\begin{array}{c}3 \\
(17.4 \%)\end{array}$ & 0 & 0 & 0 \\
\hline Anticoag & 11 & $67.6 \pm 15$ & $1640 \pm 640$ & $18 \%$ & $\begin{array}{c}5 \\
(45 \%)\end{array}$ & $9 \%$ & $335.92 \pm 247.52$ & $\begin{array}{c}2 \\
(18 \%)\end{array}$ & $6(54 \%)$ & 0 & $\begin{array}{c}5 \\
(45.4 \%)\end{array}$ & 0 & $\begin{array}{c}4 \\
(36.4 \%)\end{array}$ \\
\hline
\end{tabular}

LDH : Lactate Dehydrogenase at the time of the diagnosis; Hist emb: history of confirmed episode of renal embolism or embolism at another site; AF: evidence of atrial fibrillation at the time of the diagnosis; Valvul: Known valvular disease at the time of the diagnosis; Creat: Creatinine concentration at the time of the diagnosis; Clinical results: vascularization according to DTPA; Technical results: evidence of revascularization of the arterial territory dependent on the occluded artery. Hemato: hematoma. Hemorr: Hemorrhage. 
infarction pulmonary embolism). Nevertheless, it is not a specific marker because increases also occur in various situations involving cellular lesion, such as hemolysis, rhabdomyolysis and acute hepatitis. In renal infarction, LDH concentration reaches maximum levels a few hours after the acute episode and then follows a decreasing curve, remaining elevated during 3 to 7 days [12]. The maximum LDH value depends on the size of the renal infarction and the time since onset; hence, it is difficult to define the $\mathrm{LDH}$ concentration with the greatest sensitivity and specificity for the diagnosis of renal embolism. Very high values can lead to numerous false-negatives results and low values to false-positives $[9,11]$. In a previous study carried out by our group [9] in order to validate an algorithm based on emergency LDH determination and presence of clinical risk factors for RAE, we observed that the cut-off value for LDH with the highest sensitivity and specificity for screening purposes was $>600$ $\mathrm{U} / \mathrm{L}$. Even though this value is clearly lower than that observed in the acute phase of renal embolism, it has some important advantages. When it is associated with atypical abdominal or low back pain, few false-positive results are produced, and it allows identification of patients in a later phase when LDH levels are decreasing, as well as patients who experience smaller infarctions. It is important to note that an elevated percentage of patients included in the present study had been seen for atypical low back or abdominal pain once or several times in other centers during the same episode. In these cases, the use of a routine algorithm in the emergency room led to the diagnosis of RAE even after a lengthy delay. This delay also explains why the mean time between the onset of symptoms and the start of a therapeutic intervention was so long in our series.

The second relevant aspect of our study refers to the analysis of treatment outcome. Currently, there is no agreement as to the optimum treatment $[13,14]$. The reported therapeutic interventions are difficult to assess because most of the studies are retrospective and there are no controlled trials comparing the various available options [4,6-8]. In intrarenal embolism there is no consensus regarding the indications, dose and duration of fibrinolytic treatment, and in main renal artery embolism, some authors suggest surgical embolectomy [15], whereas others have described favorable results with local fibrinolytic treatment $[16,17]$.

Even though we used essentially arbitrary predetermined criteria to establish each of the treatment indications, the present study has the advantage that all the patients included were treated according to the same protocol; hence, the outcome achieved with each of the therapeutic interventions could be analyzed in a more homogeneous manner. On the other hand, we recognize its limitations in that it is an uncontrolled study and consequently no definitive conclusions regarding different treatment options can be reached.

In the cases of embolism of the main renal artery, revascularization was achieved in all the patients who underwent surgical embolectomy, whereas only partial revascularization was attained in 2 of the 4 patients treated with local fibrinolytics because of contraindications for surgery. In these 4 patients, fibrinolytic perfusion had to be maintained for more than 48 hours because a favorable response had not been achieved at 24 or 36 hours, and in 3 patients the therapy was discontinued because bleeding complications had developed.
In the light of these results, we believe that embolectomy should be the treatment of choice in patients with embolism of the main renal artery with a solitary functioning kidney and no surgical contraindications because it provides a better technical outcome and shortens the period of renal ischemia. Successful revascularizations have been described in patients with a prolonged time of embolism $[18,19]$. This is attributed to partial irrigation of the renal parenchyma by collateral circulation (ureteral, suprarenal or perirenal arteries) or the fact that the occlusion was not complete. When renal flow is re-established in the absence of massive infarction, renal function can improve once the concomitant tubular failure has resolved. For this reason it is difficult to establish a maximum period of time after which surgery is not recommendable.

Local perfusion of fibrinolytics is being used with increasing frequency and has yielded satisfactory results. Although some authors have suggested that fibrinolytic treatment should be used as the first treatment of choice in all cases of renal embolism [4], there is no consensus as to the indications or the duration of this therapy. In our experience with the local fibrinolysis regimen utilized, technical revascularization was achieved in all patients with intrarenal embolism in less than 36 hours and without evidence of bleeding complications. Therefore, it can be concluded that for patients with intrarenal embolism and no absolute contraindications, this therapy is effective and safe, and is likely to be preferable to conservative treatment with anticoagulation, since it is associated with a better technical outcome and no increase in mortality.

Systemic anticoagulation has been widely applied to treat RAE. Although its use as an effective measure for thrombus lysis is formally questionable, it allows physiological fibrinolysis and prevents subsequent embolic episodes [20]. Thus, we believe it should be used in all patients with contraindications for the other treatment options, but not for anticoagulation.

Definite mortality rates for each type of treatment are not available in the literature; however, the overall mortality observed in our series is within the values described by other authors [4]. Because of their elevated comorbidity, mortality was higher in the group of patients who could not undergo surgery or fibrinolytic therapy, as compared to the remaining patients.

Other published studies all include a small number of patients ranging from 11 cases [7], 17 cases [4, 6], and the most recent, 44 cases [8], all of a retrospective nature in which the clinical background of patients is analysed, the RAE diagnosis appearing in these patients' discharge report or in diagnosis made through necropsic findings. Hazanov's study [8], the most comprehensive of the four, published in 2004 in Medicine, with 44 cases studied retrospectively, also contains a thorough review of the other three studies mentioned. It should be pointed out that there are many other publications about isolated cases, both descriptive as well as on aspects of treatment by surgery, with fibrinolytics or with anticoagulants, but being isolated cases or at most describing two or three patients, no conclusions can be reached [13,2127]. When we compare the characteristics of patients with RAE in our prospective study with the other four retrospective studies and the total 130 patients (Table 4), we can es- 
Table 4. Demographic, Clinical, Analytical Data and Patients' Evolution from the Main Studies

\begin{tabular}{|c|c|c|c|c|c|c|c|c|c|c|c|}
\hline Lessman 1978 & 17 & 58 & $12 / 5$ & 4 & 13 & 9 & 15 & 14 & 12 & $\begin{array}{c}4 \\
23.5 \%\end{array}$ & $\begin{array}{c}4 \\
23.5 \%\end{array}$ \\
\hline Domanovits 1999 & 17 & 68 & $8 / 9$ & 6 & 17 & 2 & 6 & 14 & 4 & 0 & 0 \\
\hline Korzets 2002 & 11 & 67.4 & $5 / 5$ & 3 & 10 & 0 & 3 & 9 & 7 & $\begin{array}{c}1 \\
10 \%\end{array}$ & 0 \\
\hline Fort 2007 & 41 & 66.2 & $15 / 26$ & 10 & 35 & 15 & 19 & 41 & 30 & $\begin{array}{c}8 \\
19.5 \%\end{array}$ & $\begin{array}{c}6 \\
14.6 \%\end{array}$ \\
\hline Total & 130 & 65.8 & $62 / 68$ & 30 & 116 & 29 & 66 & 119 & 72 & $\begin{array}{c}16 \\
13 \%\end{array}$ & $\begin{array}{c}15 \\
11.6 \%\end{array}$ \\
\hline
\end{tabular}

Pat: Patients. Prev embo: previous embolisms. Olig: oliguria. Creat: creatinine. Diag: diagnosis. ESRD: end stage renal disease.

tablish that the age is similar in all of them, with an average age of 65.8 , with a slight predominance of prevalence in women and $23-24 \%$ of patients with previous episodes of embolism. Lumbar pain is common in all patients $(89.2 \%)$ with no differences between the studies. Renal insufficiency, defined by creatinine equal to or above $132,6 \mu \mathrm{mol} / 1$ was found in $50.7 \%$ of all patients. The percentage of patients with renal insufficiency in our study was similar to that of Hazanov [8], with Lessman's study [4] showing a greater percentage of patients with oliguria and/or renal insufficiency. We found a greater percentage of patients with oliguria, probably resulting from the large number of patients with a single functioning kidney in our study. Regarding LDH figures we found a high level in all our patients in our study since it was a prospective study based on diagnosis by means of an increase in LDH. However, the other studies also showed an increase in $90.6 \%$ of patients, which reinforces the usefulness of LDH in diagnosing RAE. Our study, together with that of Lessman [4], was the one that showed the greatest number of patients with end-stage chronic renal disease, with the total average of all studies being $13 \%$. Mortality in our study was similar to that of the other studies, on the whole around $11.6 \%$. Mention should be made of the greater percentage of mortality in Lessman's study [4] and the absence of mortality in the studies by Domanovits [6] and Korzets [7] (Table 4).

\section{CONCLUSIONS}

The data from our cohort confirms that the use of a diagnostic algorithm based on serum LDH levels determined on an emergency basis is useful for identifying renal embolism patients who present an atypical clinical picture.

Despite the obvious limitations of an uncontrolled study, we believe that until randomized clinical trials are available comparing the results of embolectomy versus fibrinolysis in main renal artery embolism and local fibrinolysis versus systemic anticoagulation in intrarenal embolism, we suggest surgical embolectomy for patients with main renal artery embolism and no surgical contraindications, since it can provide the best technical outcome in the shortest time possible. In the case of intrarenal embolism, we believe that the indication for local fibrinolysis or anticoagulation should be de- termined by the extent of the embolism and the severity of renal failure. In patients with a single kidney or multiple intrarenal embolisms, local fibrinolytic treatment is likely to shorten the duration of renal ischemia and preserve renal parenchyma; hence it should be the treatment of choice in this population. In cases of unilateral intrarenal embolism without compromise of renal function, the choice of treatment is complex, since the risk associated with intrarenal perfusion of fibrinolytics may surpass the potential benefit obtained with revascularization.

\section{ABBREVIATIONS}

$\begin{array}{lll}\mathrm{RAE} & = & \text { Renal Artery Embolism } \\ \mathrm{LDH} & = & \text { Lactate Dehydrogenase } \\ \mathrm{CT} \text { angiography } & = & \text { Computed Tomography }\end{array}$

\section{REFERENCES}

[1] Hoxie HJ, Coggin CB. Renal infarction. Statistical study of two hundred and five cases and detailed report of an unusual case. Arch Int Med 1940; 65: 587-94.

[2] Gleen JF, Boyce WH, Kaufman JJ. Salvat Inc. Eds. Cirugía Urológica, Barcelona: 1986; 297.

[3] Traube L. Uber deu Zusammenhaug von Herz und Nierenkrankheit. Berlin, A. Hirschwald, 1856; 77.

[4] Lessman RK, Johnson SF, Coburn JW, et al. Renal artery embolism: clinical features and long-term follow-up of 17 cases. Ann Inter Med 1978; 89: 477-82.

[5] Fort J, Segarra A, Camps J, et al. Embolismo de arteria renal como causa de fracaso renal agudo. Diagnóstico, factores pronósticos y tratamiento. Nefrología 1992 ; 12 (Suppl 4): S194-S198.

[6] Domanovits II, Paulis M, Nifkardjam M, et al, Acute renal infarction. Clinical characteristics of 17 patients. Medicine (Baltimore) 1999; 78: 386-94.

[7] Korzets Z, Plotkin E, Bernheim J, et al. The clinical spectrum of acute renal infarction. Isr Med Assoc J 2002; 4: 781-4.

[8] Hazanov N, Somin M, Attali M, et al. Acute renal embolism: fortyfour cases of renal infarction in patients with atrial fibrillation. Medicine (Baltimore) 2004; 83 (5): 292-9.

[9] Fort J, Segarra A, Camps J, et al. Diagnóstico precoz del embolismo de arteria renal mediante determinación de lacto deshidrogenasa en urgencias de un gran hospital. Nefrología; 1998; 18(Suppl 3): S26

[10] Hart RG. Atrial fibrillation and stroke prevention. N Engl J Med 2003; 3459: 1015-6.

[11] Winzelberg CG, Hull JP, Agar JW, et al. Elevation of serum Lactate Dehydrogenase levels in renal infarction. JAMA 1979; 242: 268-9. 
[12] Gault MH, Steiner G. Serum and urinary enzim activity after renal infarction. Arch Intern Med 1972; 129: 958.

[13] Gasparini M, Hofmann R, Stoller M. Renal artery embolism: clinical features and therapeutic options. J Urol 1992; 147: 567-72.

[14] Lacombe M. Surgical versus medical treatment of renal artery embolism. J Cardiovas Surg 1977; 18: 281-0.

[15] Boutier S, Valverde JP, Lacombe M, et al. Renal artery emboli: the role of surgical treatment. Ann Vasc Surg 1988; 2: 161-8.

[16] Blum U, Billman P, Krause T, et al. Effect of local low-dose thrombolysis on clinical outcome in patients with acute embolic renal artery occlusion. Radiology 1993; 189: 549.

[17] Rouviere O, Berger P, Beziat C, et al. Acute thrombosis of renal transplant artery: graft salvage by means of intra-arterial fibrinolysis. Transplantation 2002; 73: 403.

[18] Perkins RP, Jacobsen DS, Feder FP, et al. Retourn of renal function after late embolectomy. Report of a case. N Engl J Med 1967; 276: 1194.

[19] Fort J, Camps J, Ruíz P, et al. Renal artery embolism successfully revascularized by surgery after 5 days' anuria. Is it never too late?. Nephrol Dial Transplant 1996; 11: 1843-5.
[20] Moyer JD, Rao CN, Widrich WC, et al. Conservative management of renal artery embolus. J Urol 1973; 109: 138.

[21] Gutiérrez A, García M, González de Garay M, et al. Fibrinolisis local en el embolismo de arteria renal. An Med Interna 1999; 16 : 253-5.

[22] Boyer L, Ravel A, De Fraissinette B, et al Recanalisation percutanée d'artères rénales occluses. J Mal Vasc 2000; 25: 377-81.

[23] Cheng KL, Tseng SS, Tarng DC. Acute renal failure caused by unilateral renal artery thromboembolism. Nephrol Dial Transplant 2003; 18: 833-55.

[24] Rossique P, Moreno MJ, Martínez FJ, et al. Fibrinólisis local y angioplastia transluminal en embolismo de la arteria renal. Med Clin 1988; 91: 267-9.

[25] Marrón B, Ubeda I, Gallego J, et al. Functional renal recovery after spontaneous renal embolization in a sole kidney. Nephrol Dial Transplant 1997; 12: 2417-9.

[26] Morris D, Kisly A, Stoyka CG, et al. Spontaneous bilateral renal artery occlusion associated with chronic atrial fibrillation. Clin Nephrol 1993; 39: 257-9.

[27] Nicholas GG, DeMuth WE. Treatment of renal artery embolism. Arch Surg 1984; 119: 278-81. 